\title{
Effect of P-glycoprotein modulation with cyclosporin A on cerebrospinal fluid penetration of doxorubicin in non-human primates
}

Received: 18 May 1999 / Accepted: 3 September 1999

\begin{abstract}
Purpose: P-glycoprotein (Pgp) is a transmembrane drug efflux pump that is expressed in multidrugresistant cancer cells and in a variety of normal tissues, including brain capillary endothelial cells which comprise the blood-brain barrier. We studied the effects of the Pgp inhibitor, cyclosporin A (CsA), on the cerebrospinal fluid (CSF) penetration of the Pgp substrate, doxorubicin, in non-human primates. Methods: The animals received doxorubicin alone $(2.0 \mathrm{mg} / \mathrm{kg}$ i.v. over $60 \mathrm{~min})$ or doxorubicin $(1 \mathrm{mg} / \mathrm{kg}$ i.v. over $60 \mathrm{~min})$ and CsA (loading dose $4.0 \mathrm{mg} / \mathrm{kg}$ i.v. over $2 \mathrm{~h}$, followed by continuous infusion of $12 \mathrm{mg} / \mathrm{kg}$ per day over $48 \mathrm{~h}$ ). Plasma and CSF were collected over $48 \mathrm{~h}$ and the doxorubicin concentration was measured by reverse-phase high-pressure liquid chromatography (HPLC) with fluorescence detection (detection limit $5 \mathrm{n} M$ ). A twocompartment model was fitted to the plasma concentration-time data. Results: Pgp was demonstrated to be present in the epithelium of the choroid plexus by immunohistochemical methods, indicating that CSF drug penetration could be used as a surrogate for bloodbrain barrier penetration. Steady state whole blood CsA concentrations, which were measured with a fluorescence-polarization immunoassay (TDX) that detects both CsA and its metabolites, ranged from 551-1315 $\mu \mathrm{g} /$ 1 at $24 \mathrm{~h}$. The clearance of doxorubicin in four animals was reduced by $34 \%, 38 \%, 45 \%$ and $49 \%$ when given with CsA. The doxorubicin concentration in the CSF was $<5 \mathrm{n} M$ in all animals, both after doxorubicin alone and doxorubicin with CsA. Conclusions: The Pgp inhibitor, CsA, at a concentration that alters systemic
\end{abstract}

K.E. Warren $(\bowtie) \cdot$ M.C. Patel · C.M. McCully · F.M. Balis

Pediatric Oncology Branch,

Bldg. 10/Rm. 13N240, 10 Center Drive,

National Cancer Institute, Bethesda, MD 20892, USA

e-mail: warrenk@exchange.nih.gov

Tel.: + 1-310-4961756; Fax: + 1-301-4020575

L.M. Montuenga

Medicine Branch, National Cancer Institute,

Bethesda, MD 20892, USA clearance of doxorubicin, does not appear to significantly increase the CSF penetration of doxorubicin.

Key words Multidrug resistance $\cdot \mathrm{P}$-glycoprotein · Doxorubicin · Cyclosporin A $\cdot$ Blood-brain barrier

\section{Introduction}

The entry of drugs into the central nervous system (CNS) is restricted by the blood-brain barrier (BBB) which maintains the homeostasis of the central nervous system by controlling the passage of a variety of chemicals into and out of the brain [1]. Anatomically, the BBB comprises a single layer of specialized capillary endothelial cells that are linked by tight junctions and lack fenestrations, thereby forming a continuous physical barrier between the brain and circulating blood [2].

Brain capillary endothelial cells are also equipped with transporters, such as P-glycoprotein (Pgp), which are not typically found on capillary endothelial cells in other parts of the body. Pgp is a drug efflux pump that is present on the luminal surface of brain capillary endothelial cells $[3,4]$. It appears to play an important role in excluding xenobiotics, including a variety of anticancer drugs, from the CNS [5-8]. In Pgp-deficient mdr1a knockout mice, brain concentrations of the Pgp substrate, vinblastine, are 22-fold higher than in wild-type mice [9].

Overexpression of Pgp on the surface of cancer cells contributes to the multidrug resistance phenotype. A variety of drugs that inhibit Pgp are currently undergoing preclinical and clinical testing as modulators of multidrug resistance $[8,10,11]$. Cyclosporin $\mathrm{A}(\mathrm{CsA})$ is a potent chemosensitizing agent. CsA concentrations of 1 to $5 \mu \mathrm{g} / \mathrm{ml}$ modulate Pgp in vitro $[12,13]$, and clinical trials utilizing CsA in combination with chemotherapy have been performed [14-17]. In animal models and in the clinical trials, Pgp modulation with CsA decreases the clearance of anticancer drugs that are Pgp substrates and enhances their toxicity, presumably by inhibition of 
Pgp-dependent drug elimination pathways in normal liver [18].

Because Pgp-modulating agents used to reverse the multidrug resistance phenotype in cancers can also inhibit Pgp expressed in normal tissues, we studied the effect of CsA on the penetration of the Pgp substrate, doxorubicin, into the cerebrospinal fluid (CSF) using a non-human primate model. Enhanced CNS penetration of anticancer drugs could be beneficial in the treatment of primary and metastatic brain tumors, but it could also enhance the neurotoxicity of the anticancer drug. Therefore, it is important to study the impact of Pgpmodulating agents on the CNS pharmacology of anticancer drugs that are Pgp substrates.

\section{Materials and methods}

Immunohistochemistry

CSF drug penetration was used as a surrogate for BBB penetration in the non-human primate model. Therefore, we studied the expression of Pgp on the epithelial surface of the choroid plexus and in brain capillary endothelial cells in the brains from two nonhuman primates (Macaca mulatta) that were killed for other reasons, in order to demonstrate that Pgp was also a component of the blood-CSF barrier. Sections were cut at $5 \mu \mathrm{m}$ from formalinfixed, paraffin-embedded tissue. Immunohistochemistry was performed according to the avidin-biotin peroxidase complex method using an ELITE Vectastain kit (Vector Laboratories, Burlingame, Calif.) with biotinylated antimouse immunoglobulin. The monoclonal antibodies employed were C219 (Centocor Diagnostics, Malvern, Pa,) and JSB-1 (Caltag, Burlingame, Calif.), both of which recognize different intracytoplasmic epitopes of human Pgp, and an unconjugated, purified mouse IgG1 (Caltag) a negative control. All monoclonal antibodies were diluted in phosphatebuffered saline and $0.1 \%$ bovine serum albumin to a final concentration of $10 \mu \mathrm{g} / \mathrm{ml}$. The secondary antiserum was biotinylated goat antimouse antiserum used at a dilution of $1 / 200$ for $1 \mathrm{~h}$.

\section{Animal model}

This study was approved by the NIH Animal Care and Use Committee. Four adult rhesus monkeys (Macaca mulatta) ranging in weight from $7.1 \mathrm{~kg}$ to $12.7 \mathrm{~kg}$ were used in the pharmacokinetic studies. The animals were group-housed in accordance with the Guide for Care and Use of Laboratory Animals [19] and received water and Purina Monkey Chow ad libitum. Each animal had a 4th ventricular Pudenz catheter attached to an Ommaya reservoir for CSF sampling as previously described [20]. Blood samples were drawn through a catheter placed in the saphenous or femoral vein contralateral to the site of drug administration.

Each animal received doxorubicin (Rubex, Chiron Therapeutics, Emeryville, Calif.) alone at a dose of $2.0 \mathrm{mg} / \mathrm{kg}$ i.v. over $60 \mathrm{~min}$ (equivalent to a dose of $40 \mathrm{mg} / \mathrm{m}^{2}$ in humans), or doxorubicin at $1.0 \mathrm{mg} / \mathrm{kg}$ i.v. over $60 \mathrm{~min}$ and CsA (Sigma Chemical Company, St. Louis, Mo.) administered as a loading dose of $4.0 \mathrm{mg} / \mathrm{kg}$ i.v. over $2 \mathrm{~h}$ followed by continuous infusion of $12 \mathrm{mg} /$ $\mathrm{kg}$ per day for $48 \mathrm{~h}$. The dose of doxorubicin was reduced when administered with CsA to account for the reduced clearance of doxorubicin in the presence of Pgp inhibitors. The study followed a crossover design and the order of administration (doxorubicin alone vs the combination of doxorubicin and CsA) was randomly determined.

Plasma samples were collected prior to infusion of doxorubicin, 30 min after the start of infusion, at the end of infusion, and 5, 10, $15,30,60$, and $90 \mathrm{~min}$ and $2,3,4,6,8,10,24$ and $48 \mathrm{~h}$ after the end of the infusion. CSF samples were collected prior to infusion, $30 \mathrm{~min}$ after the start of infusion, at the end of infusion, and $0.5,1$, $1.5,2,3,4,6,8,10,24$ and $48 \mathrm{~h}$ after the end of infusion. Plasma and CSF were frozen at $-70^{\circ} \mathrm{C}$ until analysis. Blood samples were also drawn for quantification of CsA plasma concentration at steady-state $(24 \mathrm{~h})$.

\section{Doxorubicin analysis}

Doxorubicin concentration in plasma and CSF was measured using an isocratic reverse-phase HPLC method with fluorescence detection. Samples were spiked with $200 \mathrm{n} M$ daunorubicin (Sigma Chemical Co., St. Louis, Mo.) as an internal standard. Plasma samples and doxorubicin plasma standards were prepared by solidphase extraction. Varian Bond Elut $3 \mathrm{ml} \mathrm{C}_{18}$ solid-phase extraction cartridges (Varian, Harbor City, Calif.) were wetted with $1 \mathrm{ml}$ methanol and washed with $1 \mathrm{ml}$ distilled deionized water prior to application of the plasma standard or sample. After the plasma standard or sample had been applied to the cartridge, it was washed with $0.5 \mathrm{ml}$ distilled deionized water and the doxorubicin was eluted with $1 \mathrm{ml}$ of $9: 1 \mathrm{methanol} /$ formic acid $\times 2$. The cartridge eluant was evaporated to dryness under a stream of nitrogen and reconstituted in $100 \mu \mathrm{l}$ of the HPLC mobile phase. CSF samples were extracted with $5 \%$ perchloric acid.

Extracted standards and samples were injected onto a $\mu$ Bondapak phenyl column (Waters Corporation, Milford, Mass.) with a PS-GU phenyl $5 \mu \mathrm{m}$ guard column (Thomson Instrument Company, Springfield, Va.). The mobile phase consisted of 79:21 (vol: vol) $0.4 M$ ammonium formate/acetonitrile, $\mathrm{pH} 4.0$, at a flow rate of $2 \mathrm{ml} / \mathrm{min}$. The HPLC system consisted of a Waters WISP 712 automated sampler, a Waters 510 pump and a Waters 470 scanning fluorescence detector set at an excitation wavelength of $480 \mathrm{~nm}$ and an emission wavelength of $595 \mathrm{~nm}$. Analysis of the chromatograms was performed with Millenium software (Waters). The limit of detection was $5 \mathrm{n} M$, the limit of quantification was $10 \mathrm{n} M$, and the coefficient of variation was $\leq 10 \%$.

\section{Cyclosporin A}

Steady-state CsA concentrations in whole blood were measured by a commercial laboratory (SmithKline-Beecham, Owings Mills, Md.) using a fluorescence-polarization immunoassay (TDX) that detects both CsA and its metabolites.

Pharmacokinetic analysis

A two-compartment model, described by the following equations, was fitted to the plasma concentration-time data using the mathematical modeling software program, MLAB (Civilized Software, Bethesda, Md.):

$\frac{\mathrm{d} C_{\mathrm{c}}}{\mathrm{d} t}=\frac{k_{0}}{V_{\mathrm{c}}}+\frac{k_{\mathrm{pc}} \cdot X_{\mathrm{p}}}{V_{\mathrm{c}}}-k_{\mathrm{cel}} \cdot C_{\mathrm{c}}-k_{\mathrm{cp}} \cdot C_{\mathrm{c}}$

and

$\frac{\mathrm{d} X_{\mathrm{p}}}{\mathrm{d} t}=k_{\mathrm{cp}} \cdot C_{\mathrm{c}} \cdot V_{\mathrm{c}}-k_{\mathrm{pc}} \cdot X_{\mathrm{p}}$

where $C_{\mathrm{c}}$ is the concentration of drug in the central compartment at time $t ; X_{\mathrm{p}}$ is the amount of drug in the peripheral compartment; $k_{0}$ is the drug infusion rate; $V_{\mathrm{c}}$ is the volume of the central compartment; $k_{\mathrm{cel}}$ is the elimination rate constant; and $k_{\mathrm{cp}}, k_{\mathrm{pc}}$ are the rate constants for exchange of drug between the central and peripheral compartments. AUC was determined from the model parameters using the area under the fitted concentration-time curve. The degree of CSF penetration of doxorubicin was derived from the expression:

$\frac{\mathrm{AUC}_{\mathrm{CSF}}}{\mathrm{AUC}_{\text {plasma }}}$ 
where $\mathrm{AUC}_{\mathrm{CSF}}$ is the area under the CSF concentration-time curve, and $\mathrm{AUC}_{\text {plasma }}$ is the area under the plasma concentrationtime curve.

\section{Results}

Pgp was present on the epithelial cells of the choroid plexus and the endothelial cells of blood vessels in the brain by immunohistochemical staining using the monoclonal antibodies C219 and JSB-1 (Fig. 1). No

Fig. 1A-I Immunohistochemical stains demonstrating the presence of P-gp on brain capillary endothelial cells that make up the $\mathrm{BBB}$ and the epithelial surface of the choroid plexus. The monoclonal antibodies, C219 and JSB-1, which are directed to human P-gp epitopes, were used. Brown staining reveals presence of antigen (Pgp). A Cerebrum (original magnification $\times 775$ ) with control antibody. B Cerebrum (original magnification $\times 350$ ) with JSB-1 antibody showing capillary endothelial cell staining. C Cerebrum (original magnification $\times 1000$ ) with C219 antibody showing capillary endothelial cell staining. D Choroid plexus (original magnification $\times 400$ ) with control antibody. E Choroid plexus (original magnification $\times 400$ ) with JSB-1 antibody showing ependymal epithelial cell staining. F Choroid plexus (original magnification $\times 400$ ) with $\mathrm{C} 219$ antibody showing ependymal epithelial cell staining. G Choroid plexus (original magnification $\times 1150$ ) with control antibody. H Choroid plexus (original magnification $\times 1050$ ) with JSB-1 antibody showing ependymal epithelial cell staining. I Choroid plexus (original magnification $\times 1050$ ) with C219 antibody showing ependymal epithelial cell staining staining for Pgp was observed in the neurons or glial cells in the brain, consistent with the findings of previous studies [21]. No staining was observed in the endothelial cells of blood vessels in the colon, although the epithelial cells lining the colon did stain positive (not shown). The isotypic monoclonal antibody that was used as a negative control did not stain the cells of the choroid plexus. C219 stained primarily along the membrane, but also had some cytoplasmic staining, whereas staining with JSB-1 was primarily intracytoplasmic.

Doxorubicin disposition in the non-human primates was similar to that observed in humans. As shown in Fig. 2, there was an initial rapid decline in plasma concentrations during the distributive phase (mean $t_{1 / 2} \alpha, 3 \mathrm{~min}$ ) followed by a more prolonged elimination phase (mean $\mathrm{t}_{1 / 2} \beta, 12 \mathrm{~h}$ ). Pharmacokinetic model parameters from fitting the two-compartment model to the doxorubicin plasma concentration-time data for individual animals with and without CsA are shown in Table 1, and the standard pharmacokinetic parameters derived from the model parameters are shown in Table 2. The clearance of doxorubicin was reduced by a mean of $42 \%$ when doxorubicin was administered in combination with CsA, and as a result, the plasma drug exposure from doxorubicin alone at a dose of $2 \mathrm{mg} / \mathrm{kg}$ was equivalent to the exposure achieved with a doxorubicin dose of $1 \mathrm{mg} / \mathrm{kg}$ in combination with CsA (Fig. 2).
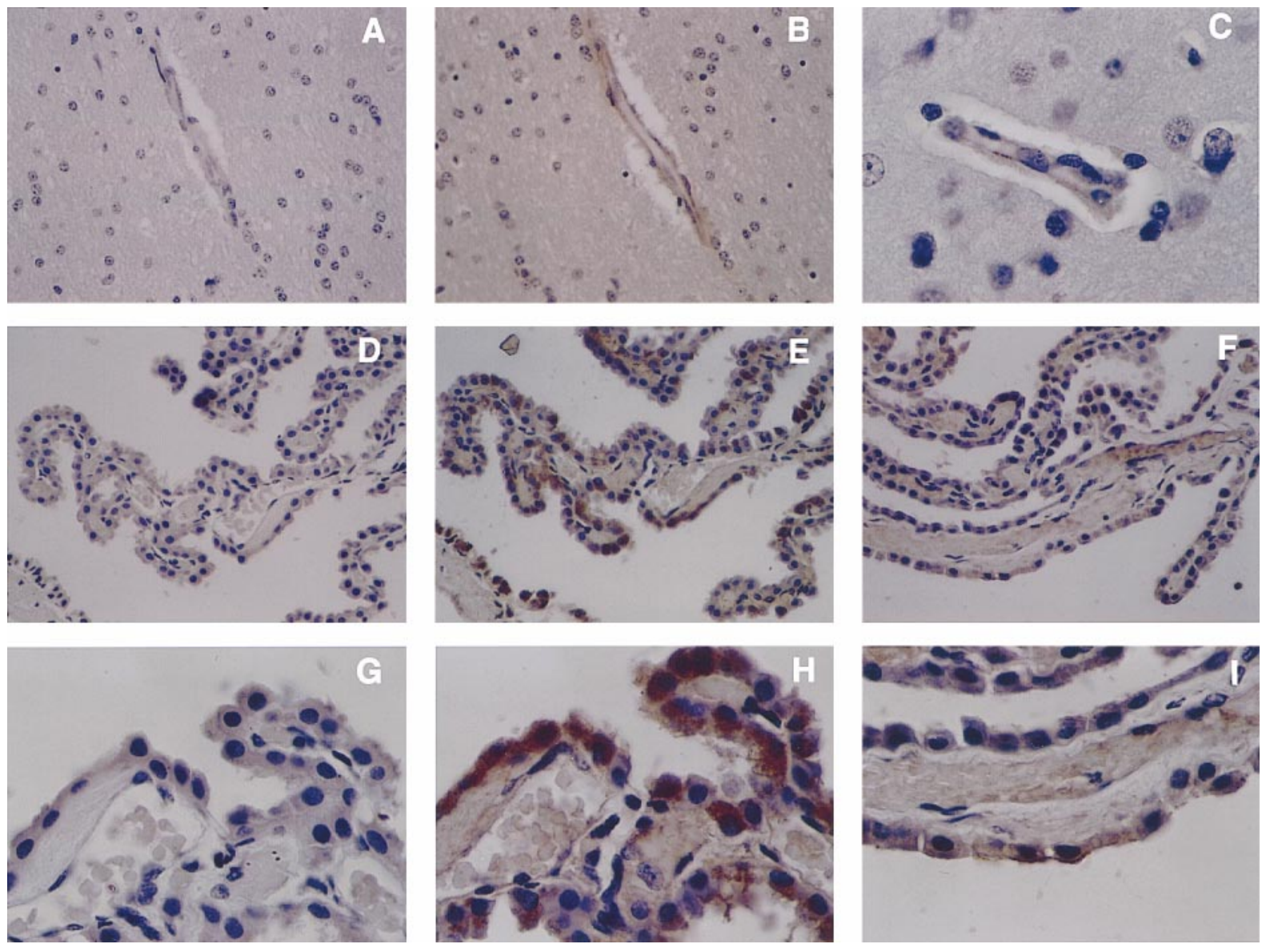


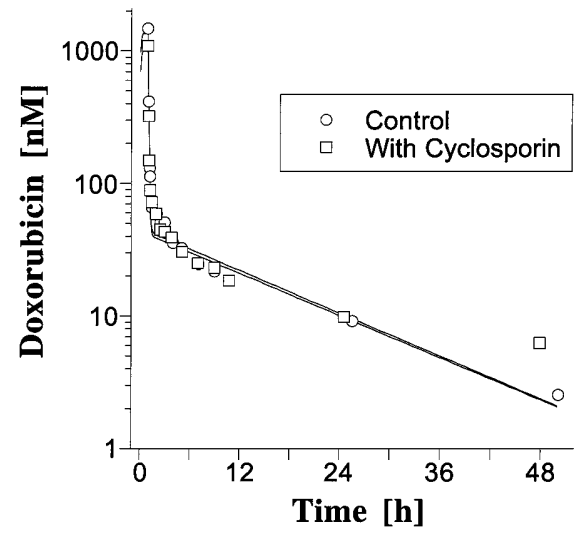

Fig. 2 Plasma concentration-time profile of doxorubicin in nonhuman primates administered alone $(O)$ at a dose of $2 \mathrm{mg} / \mathrm{kg}$ i.v. over $1 \mathrm{~h}$ and in combination with CsA $(\square)$ at a dose of $1 \mathrm{mg} / \mathrm{kg}$ i.v. over $1 \mathrm{~h}$. CsA was administered by continuous infusion $(12 \mathrm{mg} / \mathrm{kg}$ per day) over $48 \mathrm{~h}$. Points represent the mean from four animals and the lines represent the mean model fit to the concentration-time data using a two-compartment model

CsA whole blood steady-state concentrations in the four animals are shown in Table 3. There was no apparent relationship between CsA blood levels and the degree of reduction in doxorubicin clearance. There was no increase in hematologic, hepatic, renal or neurologic toxicity in animals receiving doxorubicin in combination with CsA at these doses as assessed by white blood cell nadir, serum bilirubin levels, serum creatinine levels and close observation.

Table 1 Pharmacokinetic model parameters (two-compartment model) for doxorubicin in four non-human primates that received doxorubicin ( $2 \mathrm{mg} / \mathrm{kg}$ i.v. over $60 \mathrm{~min})$ alone (-CsA) and doxor-
Table 3 Relationship between steady-state CsA concentration in blood and the reduction in the clearance of doxorubicin when doxorubicin was administered in combination with CsA

\begin{tabular}{lcl}
\hline Monkey no. & $\begin{array}{l}\text { Steady-state CsA level } \\
(\mu \mathrm{g} / \mathrm{l})\end{array}$ & $\begin{array}{l}\text { Reduction in doxorubicin } \\
\text { clearance }(\%)\end{array}$ \\
\hline R895 & 551 & 34 \\
D16 & 1315 & 38 \\
B9078 & 621 & 45 \\
R838 A & 1134 & 49 \\
\hline
\end{tabular}

The doxorubicin concentration in the CSF was below the limit of detection of the assay (i.e. $<5 \mathrm{n} M$ ) in all four animals when doxorubicin was administered alone and in combination with CsA. The CSF penetration of doxorubicin was $<5 \%$ in the absence and presence of CsA. The major doxorubicin metabolite, doxorubicinol, was also not detected in the CSF.

\section{Discussion}

In non-human primates, we detected Pgp in brain capillary endothelial cells and the epithelial cells of the choroid plexus using two monoclonal antibodies directed against different intracytoplasmic epitopes of human Pgp. C219 is an IgG2a $\kappa$ monoclonal antibody that recognizes intracytoplasmic epitopes in the region of the nucleotide binding domain found in both the mdr- 1 and mdr-3 gene products [3, 22], and JSB-1 is an IgG1 monoclonal antibody that detects a separate highly conserved epitope of Pgp [23]. Immunohisto-

\begin{tabular}{|c|c|c|c|c|c|c|c|c|}
\hline \multirow[t]{2}{*}{ Monkey no. } & \multicolumn{2}{|c|}{$V_{\mathrm{c}}(1 / \mathrm{kg})$} & \multicolumn{2}{|c|}{$k_{\text {cel }}\left(\mathrm{h}^{-1}\right)$} & \multicolumn{2}{|c|}{$k_{\mathrm{cp}}\left(\mathrm{h}^{-1}\right)$} & \multicolumn{2}{|c|}{$k_{\mathrm{pc}}\left(\mathrm{h}^{-1}\right)$} \\
\hline & $-\mathrm{CsA}$ & $+\mathrm{CsA}$ & $-\mathrm{CsA}$ & $+\mathrm{CsA}$ & $-\mathrm{CsA}$ & + CsA & -CsA & $+\mathrm{Cs} \mathrm{A}$ \\
\hline R895 & 1.8 & 1.6 & 11.1 & 8.2 & 4.9 & 3.8 & 0.12 & 0.14 \\
\hline D16 & 2.7 & 1.4 & 5.3 & 6.4 & 4.1 & 3.7 & 0.09 & 0.09 \\
\hline B9078 & 1.5 & 1.1 & 10.8 & 7.8 & 6.2 & 7.6 & 0.09 & 0.08 \\
\hline R838 A & 1.8 & 1.2 & 8.5 & 6.2 & 3.9 & 6.5 & 0.08 & 0.11 \\
\hline Mean & 2.0 & 1.3 & 8.9 & 7.2 & 4.8 & 5.4 & 0.09 & 0.10 \\
\hline SD & 0.5 & 0.2 & 2.7 & 1.0 & 1.0 & 2.0 & 0.02 & 0.03 \\
\hline
\end{tabular}

Table 2 Doxorubicin pharmacokinetic parameters derived from the model parameters (Table 1) in four non-human primates that received doxorubicin $(2 \mathrm{mg} / \mathrm{kg}$ i.v. over $60 \mathrm{~min})$ alone $(-\mathrm{CsA})$ and

ubicin $(2 \mathrm{mg} / \mathrm{kg}$ i.v. over $60 \mathrm{~min})$ with CsA administered by continuous infusion over $48 \mathrm{~h}(+\mathrm{CsA})$

\begin{tabular}{|c|c|c|c|c|c|c|c|c|}
\hline \multirow[t]{2}{*}{ Monkey no. } & \multicolumn{2}{|c|}{$V_{\mathrm{ss}}(1 / \mathrm{kg})$} & \multicolumn{2}{|c|}{$\mathrm{AUC}_{\text {plasma }}(\mathrm{n} M \cdot \mathrm{h})$} & \multicolumn{2}{|c|}{ Clearance $(1 / \mathrm{h} / \mathrm{kg})$} & \multicolumn{2}{|l|}{$t_{1 / 2}(\mathrm{~h})$} \\
\hline & $-\mathrm{CsA}$ & $+\mathrm{CsA}$ & $-\mathrm{CsA}$ & $+\mathrm{CsA}$ & $-\mathrm{CsA}$ & $+\mathrm{CsA}$ & $-\mathrm{CsA}$ & $+\mathrm{CsA}$ \\
\hline R895 & 74.2 & 46.7 & 1928 & 1444 & 20.1 & 13.2 & 8.2 & 7.5 \\
\hline D16 & 126.3 & 57.2 & 2182 & 1693 & 14.2 & 8.9 & 13.8 & 12.0 \\
\hline B9078 & 105.3 & 111.5 & 2334 & 1910 & 15.7 & 8.7 & 12.5 & 17.8 \\
\hline R838 A & 84.7 & 73.4 & 1963 & 2004 & 15.1 & 7.7 & 12.1 & 12.9 \\
\hline Mean & 97.6 & 72.2 & 2101 & 1763 & 16.3 & 9.6 & 11.6 & 12.6 \\
\hline SD & 23.1 & 28.4 & 191 & 249 & 2.6 & 2.4 & 2.4 & 4.2 \\
\hline
\end{tabular}


chemical staining for these two antibodies showed a pattern consistent with a transmembrane protein, with staining in the cytoplasm and on the plasma membrane. The presence of Pgp on the epithelial surface of the choroid plexus is further evidence that the ependymal epithelium is the site of the blood-CSF barrier and that CSF penetration is a valid surrogate for BBB penetration for studying the effects of Pgp modulators on the CNS pharmacology of anticancer drugs.

The higher brain levels of vinblastine in the mdrla knockout mouse suggests that blocking Pgp with a modulating agent such as CsA would result in higher CNS and CSF concentrations of drugs that are Pgp substrates. In our non-human primate model, we achieved CsA blood levels that exceeded concentrations required to inhibit Pgp in vitro [12, 13, 24] and resulted in altered doxorubicin pharmacokinetics $(42 \%$ reduction in doxorubicin clearance) in the animals. However, CsA did not significantly increase the CSF penetration of doxorubicin. Our results are consistent with those of a study in rodents in which high doses of the Pgp inhibitors, CsA, verapamil, amiodarone, trifluoperazine, quinidine and Bay K8644, did not increase the permeability of the BBB in mice despite altering pharmacokinetics of the cytotoxic agent in other tissues that normally express Pgp [25]. In addition, in rats CsA has no impact on brain levels of etoposide [26].

Preclinical studies using the more potent CsA analog, PSC833, have demonstrated dose-dependent CNS penetration of the drug, due to a blood concentrationdependent modulation of the BBB [27]. Clinical studies using PSC833, are ongoing, and the dose-limiting toxicity appears to be neurologic (predominantly ataxia) [28] but it has not yet been determined whether this is a direct result of Pgp inhibition or an inherent toxicity of the PSC833 that is unrelated to its inhibition of Pgp [28].

In our non-human primates, CsA did not enhance hepatic, renal, neurologic, or hematologic toxicity of doxorubicin compared to doxorubicin alone, when the drug exposure (AUC) was kept constant by lowering the doxorubicin dose in proportion to the reduction in clearance in the combination regimen.

The potential clinical consequences of effective Pgp inhibition in normal tissues that express Pgp could include enhanced chemotherapy-related toxicity due to impaired clearance of chemotherapeutics that are Pgp substrates, altered distribution of anticancer drugs with enhanced access to pharmacologic "sanctuary" sites such as the CNS and testis, and enhanced hematologic toxicity from sensitization of Pgp-expressing stem cells [28]. At the dose of doxorubicin used in this study, we observed altered doxorubicin clearance, but the CNS penetration and hematologic toxicity did not appear to be affected. The concentration of the Pgp-modulating agent that affects drug clearance may be lower than the concentration of the modulating agent that is required to open the BBB, or brain capillary endothelial cells may have other mechanisms to block drug entry into the CNS that are independent of Pgp [29-31].
Although CsA did not appear to significantly increase the CSF penetration of doxorubicin in our animal model, further studies to quantify the effects of more potent Pgp modulators on the CNS pharmacology of doxorubicin and other anticancer drugs that are Pgp substrates are warranted because of the potential impact of enhanced CNS penetration on the treatment of CNS tumors and risk for neurotoxicity.

\section{References}

1. Stewart PA, Hayakawa K, Farrell CL (1994) Quantitation of blood-brain barrier ultrastructure. Microsc Res Tech 27: 516527

2. Brightman MW (1977) Morphology of blood-brain interfaces. Exp Eye Res [Suppl] 25: 1-25

3. Thiebaut F, Tsuruo T, Hamada H, et al (1989) Immunohistochemical localization in normal tissues of different epitopes in the multidrug transport protein P170: evidence for localization in brain capillaries and crossreactivity of one antibody with a muscle protein. J Histochem Cytochem 37: 159-164

4. Cordon-Cardo C, O'Brien JP, Casals D, et al (1989) Multidrug-resistance gene (P-glycoprotein) is expressed by endothelial cells at blood-brain barrier sites. Proc Natl Acad Sci USA 86: 695-698

5. Schinkel AH, Mol CA, Wagenaar E, et al (1995) Multidrug resistance and the role of P-glycoprotein knockout mice. Eur J Cancer 31A: 1295-1298

6. Tatsuta T, Naito M, Oh-hara T, et al (1992) Physiological function of P-glycoprotein in brain capillary endothelial cells. Proc Am Assoc Cancer Res 33: 467

7. Fojo A, Akiyama S, Gottesman MM, et al (1985) Reduced drug accumulation in multiply drug-resistant human KB carcinoma cell lines. Cancer Res 45: 3002-3007

8. Willingham MC, Cornwell MM, Cardarelli CO, et al (1986) Single cell analysis of daunomycin uptake and efflux in multidrug-resistant and -sensitive KB cells: effects of verapamil and other drugs. Cancer Res 46: 5941-5946

9. Asperen J van, Schinkel AH, Beijnen JH, et al (1996) Altered pharmacokinetics of vinblastine in Mdrla P-glycoproteindeficient mice. J Natl Cancer Inst 88: 994-999

10. Gosland MP, Lum BL, Sikic BI (1990) Inhibition by modulators of drug resistance of ${ }^{3} \mathrm{H}$-vinblastine binding to plasma membranes of multidrug resistant and sensitive cells. Proc Am Assoc Cancer Res 31: 407

11. Sikic BI (1997) Pharmacologic approaches to reversing multidrug resistance. Semin Hematol 34 [Suppl 5]: 40-47

12. Osieka R, Seeber S, Pannenbacker R, et al (1986) Enhancement of etoposide-induced cytotoxicity by cyclosporin A. Cancer Chemother Pharmacol 18: 198-202

13. Slater LM, Sweet P, Stupecky M, et al (1986) Cyclosporin A reverses vincristine and daunorubicin resistance in acute lymphatic leukemia in vitro. J Clin Invest 77: 1405-1408

14. Chan H, Thorner P, Weitzmann S, et al (1992) Cyclosporin A for reversal of multidrug resistance in childhood malignancies. Proc Am Assoc Cancer Res 33: 478

15. Samuels B, Ratain M, Mick R, et al (1991) Phase I trial of multidrug resistance modulation with cyclosporin A. Proc Am Assoc Cancer Res 32: 195

16. Thiessen J, Bunting P, Bjarnason G, et al (1992) Cyclosporin A (CyA) modulation of doxorubicin (D): Pharmacokinetics, response, evaluation of the MTD. Proc Am Assoc Cancer Res 33: A2798

17. Yahanda AM, Adler KM, Fisher GM, et al (1992) Phase I trial of etoposide with cyclosporine as a modulator of multidrug resistance. J Clin Oncol 10: 1624-1634

18. Hegewisch-Becker S (1996) MDR1 reversal: criteria for clinical trials designed to overcome the multidrug resistance phenotype. Leukemia 10 [Suppl 3]: S32-S38 
19. National Research Council (1996) Guide for the Care and Use of Laboratory Animals. National Academy Press, Washington DC

20. McCully CL, Balis FM, Bacher J, et al (1990) A rhesus monkey model for continuous infusion of drugs into cerebrospinal fluid. Lab Anim Sci 40: 250-255

21. Cordon-Cardo C, O'Brien JP, Casals BD, et al (1990) Expression of the multidrug resistance gene product (P-glycoprotein) in human normal and tumor tissues. $\mathbf{J}$ Histochem Cytochem 38: 1277-1287

22. Georges E, Bradley G, Gariepy J, et al (1990) Detection of P-glycoprotein isoforms by gene specific monoclonal antibodies. Proc Natl Acad Sci U S A 87: 152-156

23. Scheper RJ, Bulte JW, Brakkee JG, et al (1988) Monoclonal antibody JSB-1 detects a highly conserved epitope on the P-glycoprotein associated with multidrug resistance. Int $\mathbf{J}$ Cancer 42: 389-394

24. Ford JM (1995) Modulators of multidrug resistance. Hematol Oncol Clin North Am 9: 337-361

25. Arboix M, Gonzalez-Paz O, Colombo T, et al (1997) Multidrug resistance-reversing agents increase vinblastine distribu- tion in normal tissues expressing the P-glycoprotein but do not enhance drug penetration in brain and testis. J Pharmacol Exp Ther 281: 1226-1230

26. Burgio DE, Gosland MP, McNamara PJ (1996) Modulation effects of cyclosporine on etoposide pharmacokinetics and CNS distribution in the rat utilizing microdialysis. Biochem Pharmacol 51: 987-992

27. Lemaire M, Bruelisauer A, Guntz P, et al (1996) Dose-dependent brain penetration of SDZ PSC 833, a novel multidrug resistance-reversing cyclosporin, in rats. Cancer Chemother Pharmacol 38: 481-486

28. Fisher GA, Sikic BI (1995) Clinical studies with modulators of drug resistance. Hematol Oncol Clin North Am 9: 363-382

29. Tew K (1994) Glutathione-associated enzymes in anticancer drug resistance. Cancer Res 54: 4313-4320

30. Nooter K, Sloter G (1996) Molecular mechanisms of multidrug resistance in cancer chemotherapy. Path Res Pract 192: 768780

31. Cole SPC, Bhardwaj G, Gerlach JH, et al (1992) Overexpression of a transporter gene in a multidrug-resistant human lung cancer cell line. Science 258: 1650-1654 